${ }^{2}$ School of Biotechnology and

Biomolecular Sciences and Centre for Marine Biofouling and Bio-innovation, University of New South Wales, Sydney, NSW 2052, Australia

Correspondence: Sylvia M. Kirov (S.M.Kirov@utas.edu.au)

Purevdorj-Gage, B., Costerton, W. J. \& Stoodley, P. (2005). Phenotypic differentiation and seeding dispersal in non-mucoid and mucoid Pseudomonas aeruginosa biofilms. Microbiology 145, 1569-1576.

Webb, J. S., Thompson, L. S., James, S., Charlton, T., Tolker-Nielsen, T., Koch, B., Givskov, M. \& Kjelleberg, S. (2003). Cell death in Pseudomonas aeruginosa biofilm development. J Bacteriol 185, 4585-4592.

Webb, J. S., Lau, M. \& Kjelleberg, S. (2004). Bacteriophage and phenotypic variation in Pseudomonas aeruginosa biofilm development. J Bacteriol 186, 8066-8073.

DOI 10.1099/mic.0.28295-0

\section{Clinical significance of seeding dispersal in biofilms: a response}

We welcome the dialogue concerning the potential clinical significance of seeding dispersal (Purevdorj-Gage et al., 2005) in the life cycle of mucoid Pseudomonas aeruginosa biofilms. We had based our hypothesis that the seeding dispersal phenomenon may be more relevant for non-mucoid, environmental strains on (1) the propensity of diseases associated with reduced mucociliary clearance in the lung, such as cystic fibrosis (CF) or chronic obstructive pulmonary disease (COPD), to select for mucoid $P$. aeruginosa phenotypes, and (2) that conversion to mucoidy is usually associated with a concomitant down-regulation of flagella production and loss of swimming motility (see Garrett et al., 1999). However, as Kirov et al. discuss above, there may be greater diversity in mucoid CF isolates than generally acknowledged, and the perceived dichotomy between mucoidy and swimming phenotypes should be a topic for debate. More recently it has been shown that expression of flagellum genes in response to oxygen limitation precedes loss of mucoidy and is reversible during this state of transition (Wyckoff et al., 2002). Further, in an ongoing screen of mucoid CF isolates it was found that 6 out of 20 were motile (D. J. Wozniak, personal

communication). However, it was suspected that growth medium may also play a role in the outcome of the motility assay and exact proportions may vary depending on culture conditions. The growth-condition-dependent transient switching between mucoid and swimming phenotypes is problematic when relating a particular biofilm behaviour with phenotype and stresses the importance of attempting to characterize the phenotypic state at various time points during biofilm development. Broadly though, the finding of Dr Wozniak is in agreement with that of Kirov et al. We agree that to fully assess the role of seeding dispersal (and other yet unidentified behavioural developmental phenotypes) in the context of lung infections, biofilm studies should include greater diversity in strains, growth conditions and be conducted over longer time scales. The interesting observation that seeding motility occurred in a mucoid CF isolate clearly demonstrates that the phenomenon may have clinical relevance.

Another point we would like to briefly address is the difference in terminology used by our two groups in reference to the hollowing out of clusters. Kirov et al. refer to hollowing resulting from 'bacteriophagemediated lysis' as 'death-associated dispersal'. We use the term 'seeding dispersal' in a more general sense to describe sequential steps from the formation of microcolonies culminating in the release of live cells from the centres of the colonies. Our working hypothesis for seeding dispersal which integrates observations from our two groups is thus: (1) the formation of cell clusters; (2) the differentiation of the cell cluster into two or possibly three distinct phenotypes consisting of the cells in the stationary cluster 'wall', the highly motile seeding subpopulation in the interior and the lysing interior subpopulation (Webb et al., 2003) which allows the interior to liquefy; (3) highly agitated 'seeding motility' in the interior; and finally (4) the swimming, or swarming, of cells out of the microcolony. In the case of PAO1 the release appears to occur through directed motility, but we recognize that hollowing in non-motile species such as Staphylococcus epidermidis ( $\mathrm{P}$. Stewart \& B. Pitts, personal communication) and Actinobacillus actinomycetemcomitans (Kaplan et al., 2003) may be driven by water currents. It is interesting to speculate that the recent increase in reports of hollow clusters occurring in a diversity of bacterial biofilms is evidence of convergent evolution in biofilm development.

\section{P. Stoodley, ${ }^{1}$ B. Purevdorj-Gage ${ }^{2} \dagger$ and J. W. Costerton ${ }^{3}$}

${ }^{1}$ Center for Genomic Sciences, Allegheny-Singer Research Institute, Pittsburgh, USA

${ }^{2}$ Center for Biofilm Engineering and Dept of Microbiology, Montana State University-Bozeman, MT 59717, USA

${ }^{3}$ University of Southern California, Center For Biofilms, Los Angeles, CA, USA

†Present address: Division of Health Sciences, Montana State UniversityBozeman, MT 59717, USA

Correspondence: B. Purevdorj-Gage

(laura_p@erc.montana.edu)

Garrett, E. S., Perlegas, D. \& Wozniak, D. J. (1999). Negative control of flagellum synthesis in Pseudomonas aeruginosa is modulated by the alternative sigma factor $\mathrm{AlgT}$ (AlgU). J Bacteriol 181, 7401-7404.

Kaplan, J. B., Meyenhofer, M. F. \& Fine, D. H. (2003). Biofilm growth and detachment of Actinobacillus actinomycetemcomitans. J Bacteriol 185, 1399-1404.

Purevdorj-Gage, B., Costerton, W. J. \& Stoodley, P. (2005). Phenotypic differentiation and seeding dispersal in non-mucoid and mucoid Pseudomonas aeruginosa biofilms. Microbiology 145, 1569-1576.

Webb, J. S., Thompson, L. S., James, S., Charlton, T., Tolker-Nielsen, T., Koch, B., Givskov, M. \& Kjelleberg, S. (2003). Cell death in Pseudomonas aeruginosa biofilm development. J Bacteriol 185, 4585-4592.

Wyckoff, T. J. O., Thomas, B., Hassett, D. J. \& Wozniak, D. J. (2002). Static growth of mucoid Pseudomonas aeruginosa selects for non-mucoid variants that have acquired flagellum-dependent motility. Microbiology 148, 3423-3430.

DOI 10.1099/mic.0.28456-0 